\title{
REASENTAMIENTOS: UNA MIRADA CONCEPTUAL DESDE EL ORDENAMIENTO TERRITORIAL Y LA GESTIÓN DE RIESGOS
}

\author{
Galo Ernesto Grijalva May ${ }^{1 \star}$, Henry Antonio Pacheco Gil ${ }^{2}$, Rosa Maricela Cedeño Zambrano ${ }^{3}$ \\ 'Instituto de Postgrado de la Universidad Técnica de Manabí, Portoviejo, Ecuador \\ ¿Universidad Técnica de Manabí, Portoviejo, Ecuador. \\ 3 Universidad Técnica de Manabí, Portoviejo, Ecuador. \\ *Autor para correspondencia: galogrijalvamay@gmail.com \\ Recibido: 2021/02/12 \\ Aprobado: 2021/06/04 \\ D0l: https://doi.org/10.26621/ra.v1i24.613
}

\section{RESUMEN}

El ser humano, a lo largo de la historia, se ha caracterizado por su relación agresiva con el medio físico que lo rodea, lo que ha dado lugar al surgimiento de zonas de riesgos que generan consecuencias significativas como los procesos de reasentamiento poblacional. El presente estudio tiene como objetivo realizar un análisis documental sobre los reasentamientos poblacionales que se producen como consecuencia de las políticas del ordenamiento territorial (OT) y Gestión de Riesgos. Para ello, se realizó una revisión sistemática exploratoria de documentación científica que permitió seleccionar la información pertinente de acuerdo a las variables de estudio. Se logró reunir el criterio de varios autores, los cuales argumentan que los reasentamientos resultan físicamente pertinentes, pues, al modificar la localización de la población vulnerable, se elimina el componente exposición al no existir coincidencia espacio-temporal con la amenaza natural, lo que deviene en la anulación de la condición de riesgo, al tiempo que contribuye a la regeneración ambiental. Sin embargo, como resultado de estos procesos, se encontraron hallazgos como afectaciones severas a la economía familiar y al sentido de identidad y pertenencia en los nuevos asentamientos, además de situaciones de exclusión social y desarraigo que, en la mayoría de los casos, no son tratados psicológicamente.

Palabras clave: Apego al hábitat; desarrollo social; gestión de riesgos; ordenación del territorio, reasentamiento humano.

\section{ABSTRACT}

The human being throughout history has been characterized by its aggressive relationship with the physical environment that surrounds it, which has led to the emergence of risk areas that It generates significant consequences such as population resettlement processes. The objective of this study is to carry out a documentary analysis on the population resettlements that occur as a consequence of land use planning (OT) and Risk Management policies, for which a systematic exploratory review of scientific documentation was carried out that allowed the selection of relevant information according to the study variables. It was possible to meet the criteria of several authors, who argue that resettlements are physically relevant, since by modifying the location of the vulnerable population, the exposure component is eliminated as there is no spatio-temporal coincidence with the natural hazard, which results in the cancellation of the risk condition, while It is contributing to environmental regeneration, however, as a result of these processes, findings were found such as severe effects on the family economy and the sense of identity and belonging in the new settlements, in addition to social situations exclusion and uprooting that in most cases are not treated psychologically. Keywords: Habitat attachment; human resettlement; risk management; social development; spatial planning.

Keywords: Habitat attachment; human resettlement; risk management; social development; spatial planning. 


\section{INTRODUCCIÓN}

Según datos de la ONU - Hábitat (2016), el 54\% de la población mundial habita en las zonas urbanas, mientras que la mayor parte de esta población vive en ciudades intermedias. Esto ha provocado que los desastres, de origen natural o humano, produzcan cada vez mayores impactos en las condiciones de vida de las personas, lo que conlleva a la aplicación de mecanismos de prevención de riesgos como son los reasentamientos poblacionales, que, en los casos estudiados, forman parte de las políticas del ordenamiento territorial de las localidades.

Mejía-Escalante (2012) hace un aporte conceptual significativo; propone que en un reasentamiento de población están implícitos el territorio que se desaloja, o hábitat origen; y el lugar de llegada, o hábitat destino. Esto permite establecer los cambios que sufre la población reasentada, la cual, por una parte, se beneficia por el patrimonio, seguridad y los servicios públicos del nuevo emplazamiento, mientras que, por otra, se ve afectado económicamente al incrementarse ciertos gastos como en transporte y movilidad al trabajo y centros de estudio, impuestos a la vivienda, permanencia, entre otros.

Otro aspecto destacado ocurre en las esferas culturales y sociales, en donde existen conflictos derivados del arraigo a las costumbres y el apego al hábitat de origen; esto se da por las normas de convivencia social existentes en el nuevo emplazamiento, las cuales, en algunos casos, llegan a ser totalmente distintas, provocando sentimientos de desapego al nuevo hábitat.

Bajo este precedente, se tiene por objetivo realizar el análisis documental de estudios sobre reasentamientos poblacionales como resultado de las políticas del ordenamiento territorial para la gestión de riesgos de zonas de alto riesgo no mitigable, o en el marco de catástrofes naturales, generalmente ocasionados por la intervención humana en espacios no destinados para el hábitat, sin dejar de lado las implicaciones socio culturales y emocionales de las personas que experimentan estos procesos.

\section{MÉTODOS}

El presente estudio se desarrolló a partir de un análisis documental de los reasentamientos poblacionales como efectos del ordenamiento territorial para la prevención de desastres y gestión de riesgos, retomando la experiencia de otros autores, a través de estudios de posgrado, artículos científicos, libros y publicaciones del tema. Se llevó a cabo una revisión sistemática exploratoria y, siguiendo a lo propuesto por Orsini, Danús \& Tricio (2019), se definieron los criterios de inclusión y exclusión (ver tabla 1).

Posterior a ello se aplicó el protocolo de la Revisión Sistemática Exploratoria, el cual consiste en: 1) Búsqueda y selección de la información; 2) Organización de la información y; 3) Análisis de la información. Todo ello es previo a la presentación de resultados y la discusión.

\section{Búsqueda y selección de la información}

Para la búsqueda preliminar se empleó Google Académico; de este proceso, emergieron 338 documentos en castellano y 834 documentos en inglés, a partir de la revisión de las palabras clave: Apego al hábitat; Desarrollo social; Gestión de riesgos; Ordenación del territorio, Reasentamiento humano. En la exploración efectuada se escogieron un total de 81 artículos relacionados con los reasentamientos poblacionales ocasionados por desastres o amenazas de origen natural. Siguiendo con el postulado de López (2011), para seleccionar la información se aplicaron parámetros de inclusión como documentaciones en idioma castellano e inglés, en un periodo comprendido entre 2010-2020, de las bases de datos científicas Elsevier; Redalyc; Scopus y SciELO. Se estableció como parámetro de exclusión a la literatura que no aporta de manera significativa a la investigación. Para el diseño de la metodología también se tomó en cuenta el análisis de contenido, para lo cual se evaluaron las siguientes variables:

- Reasentamientos: Ordenamiento Territorial y Desarrollo.

- El apego a la vivienda y al lugar de origen.

- El Reasentamiento como mecanismo de Gestión de Riesgos.

De los 81 artículos preseleccionados, se excluyeron 46 ya que estos no superaron los criterios de inclusión enfocados en las variables ya descritas. De los 35 artículos seleccionados, 7 fueron publicados en idioma inglés (20\%) y 31 en castellano (80 \%).

Tabla 1. Criterios de Inclusión y Exclusión

\begin{tabular}{lll}
\hline & Criterios de Inclusión & \multicolumn{1}{c}{ Criterios de Exclusión } \\
\hline $1 \quad \begin{array}{l}\text { Estudios enfocados en } 1 \\
\text { reasentamientos de po- } \\
\text { blación que incluyan cau- } \\
\text { sas como desastres de } \\
\text { origen natural o antrópico }\end{array}$ & $\begin{array}{l}\text { Estudios enfocados en reasenta- } \\
\text { mientos voluntarios por causas no } \\
\text { asociadas a riesgos y/o desastres }\end{array}$ \\
\hline $2 \quad \begin{array}{ll}\text { Estudios enfocados en 2 } \\
\text { reasentamientos de po- } \\
\text { blación como efectos de } \\
\text { procesos de ordenación } \\
\text { del territorio }\end{array}$ & $\begin{array}{l}\text { Estudios enfocados en reasenta- } \\
\text { mientos voluntarios por causas no } \\
\text { ción del territorio }\end{array}$ \\
\hline
\end{tabular}

3 Estudios empíricos o pri- $3 \quad$ Estudios publicados en idiomas dimarios ferentes al español o inglés.

4 Estudios publicados en 4 Estudios cuantitativos o cualitativos idioma español 0 inglés. que no aportan definiciones 0 análisis claros sobre reasentamientos poblacionales

5 Estudios cuantitativos 05 Publicaciones en revistas no indicualitativos con adecuazadas en bases de datos científicas das definiciones, análisis de datos y conclusiones válidas

\section{Organización de la información}

Los documentos seleccionados se organizan de forma sistemática por relevancia, distinguiendo los principales documentos de los secundarios. Se elaboró una matriz para el análisis bibliométrico de cada una de las publicaciones, incorporando los siguientes ítems: autores; año; tema; país; enunciado principal; objetivo; método; resultados-conclusiones; esto, nos permitió proceder a la revisión sistemática enfocada en los reasentamientos motivados por eventos adversos de origen natural 0 antrópico.

Los artículos incluidos en la investigación fueron organizados geográficamente, dando como resultado lo presentado en la Tabla N.2. 
Tabla 2. Ubicación geográfica de los artículos revisados

\begin{tabular}{lcc}
\hline \multicolumn{1}{c}{ Artículos por país } & Cantidad & Porcentaje \\
Colombia & 12 & 34,29 \\
\hline Ecuador & 4 & 11,43 \\
\hline Chile & 4 & 11,43 \\
\hline España & 1 & 2,86 \\
\hline Argentina & 2 & 5,71 \\
\hline EEUU & 2 & 5,71 \\
\hline Reino Unido & 2 & 5,71 \\
\hline México & 2 & 5,71 \\
\hline Kenia & 1 & 2,86 \\
\hline Holanda & 2 & 5,71 \\
\hline Perú & 1 & 2,86 \\
\hline Alemania & 1 & 2,86 \\
\hline Australia & 1 & 2,86 \\
\hline TOTAL & 35 & 100 \\
\hline
\end{tabular}

Así podemos apreciar que la información proviene de fuentes científicas fiables, de autores expertos con trayectoria académica en la temática, de 13 países distintos.

\section{Análisis de la información}

En esta etapa, se realiza un análisis de la información que previamente fue organizada, para recabar los aportes teóricos más relevantes para el estudio de los reasentamientos. Se decide el aporte del trabajo, basado en un pensamiento crítico, contrastando con criterios de diferentes fuentes bibliográficas y la experiencia de los autores.

Estos documentos fueron agrupados según las variables de estudio, en la siguiente tabla:

Tabla 3. Autores por variable de estudio

\begin{tabular}{|c|c|c|c|}
\hline $\begin{array}{l}\text { Variables de } \\
\text { estudio }\end{array}$ & Autores y año de publicación & No & $\%$ \\
\hline $\begin{array}{l}\text { Ordenamiento } \\
\text { Territorial y } \\
\text { desarrollo } \\
\text { social }\end{array}$ & $\begin{array}{l}\text { (Castiblanco et al., 2019); (Iturralde, 2019); } \\
\text { (Galarza, 2019); (Nikuze et al., 2019); } \\
\text { (Baum, 2015); (Duriez, 2019); (Codosero } \\
\text { et al., 2020); (Engelman, 2013); (Noorloos } \\
\text { y Kloosterboer, 2017) }\end{array}$ & 9 & 25,71 \\
\hline $\begin{array}{l}\text { El hábitat, la } \\
\text { vivienda y el } \\
\text { apego al lugar } \\
\text { de origen }\end{array}$ & $\begin{array}{l}\text { (Hábitat, 2016); (Chardon \& Hurtado, } \\
\text { 2012); (Osorio, 2017); (Chenut \& Ocam- } \\
\text { po, 2014); (Castro \& Vélez, 2018); (Me- } \\
\text { jía-Escalante, 2012); (Osorio, Maya \& } \\
\text { Rojas, 2015); (Orozco \& Guzmán; 2015); } \\
\text { (Barreto, Benítez, Puntel, 2015); (Berroeta } \\
\text { et al., 2017); (Ramos, 2018); (Carvalho \& } \\
\text { Cornejo, 2018); (Morales et al., 2017); } \\
\text { (Fainstein, 2018) }\end{array}$ & 14 & 40,00 \\
\hline $\begin{array}{l}\text { Reasentamien- } \\
\text { tos, Prevención } \\
\text { y Gestión de } \\
\text { Riesgos }\end{array}$ & $\begin{array}{l}\text { (Briones, 2010); (Correa et al., 2011); (Ve- } \\
\text { lásquez, 2016); (Sridarrana et al., 2018); } \\
\text { (Berroeta, Carvalho, Di Masso, 2016); } \\
\text { (Bravo, 2017); (Klopfer, 2015); (Ramírez, } \\
\text { 2018); (Ramos, 2017); (Castro, 2017); } \\
\text { (Gomersall, 2018); (Takano, 2018); }\end{array}$ & 12 & 34,29 \\
\hline
\end{tabular}

\section{RESULTADOS Y DISCUSIÓN}

Los resultados y discusiones se presentan en tres categorías: Los Reasentamientos: Ordenamiento Territorial y Desarrollo; El apego a la vivienda y al lugar de origen y; El reasentamiento como mecanismo de Gestión de Riesgos.

\section{Los reasentamientos: ordenamiento territorial y desarrollo}

De los diferentes intentos por conceptualizar al desarrollo sostenible, surge el informe Brundtland de la Comisión Mundial de Medio Ambiente y Desarrollo, definiéndolo como aquel que permite satisfacer las necesidades de la presente generación sin comprometer la capacidad de las generaciones futuras para satisfacer las suyas (CMMAD, 1988).

Partimos de este concepto para entender la importancia del ordenamiento territorial en los proyectos de desarrollo en las localidades, lo que para Iturralde (2019) consistiría en erradicar la pobreza, fomentar la equidad, e incrementar las capacidades y libertades humanas, sin vulnerar el ambiente. Es precisamente en este último punto que se hace hincapié, dado que, según el postulado de Osorio (2017), las "políticas públicas de desarrollo urbano requieren propuestas con un enfoque en derechos humanos, que permitan enfrentar los problemas que se derivan de los procesos acelerados de urbanización", postura que no se cumple en la mayoría de los casos estudiados.

Una vez conceptualizado el desarrollo, es necesario abordar el ordenamiento territorial. Para Galarza (2019), este consiste en ordenar el territorio con base a su potencial y expectativas de la población, con desarrollo normativo propio, para poder vivir en armonía y autonomía, organizarse socialmente y acceder al uso de los recursos naturales para una distribución de los beneficios generados.

Producto de los problemas generalizados en la planificación del territorio y las políticas públicas de los países, se genera la ocupación en áreas de alto riesgo no mitigable con probabilidad de desastres, pérdidas económicas y de vidas; para ello, algunos territorios han optado como medida el reasentamiento, generando un cambio del uso del suelo y promoviendo proyectos de mejoras sociales en la calidad de vida de las personas (Baum, 2015). Sin embargo, no se ha prestado la debida atención a los vínculos afectivos de los habitantes con el nuevo hábitat.

Castiblanco et al. (2019) afirman que, para que el reasentamiento incida de manera positiva en la mejora de las condiciones de calidad urbana y de la vivienda, debe existir participación entre la empresa privada, la pública y la sociedad

Siguiendo esta lógica, Codosero et al., (2020) indican que la tierra alcanza su máximo valor cuando está totalmente urbanizada, lo que impulsa el desarrollo de proyectos inmobiliarios. Para Engelman (2013), contener el crecimiento urbano continuo e indiscriminado es uno de los principios fundamentales del desarrollo territorial y urbano sostenible, mientras que para Duriez (2019) los territorios corresponden a una lógica de producción y de reproducción.

Nikuze et al. (2019) comparten que muchos reasentamientos respaldados por políticas de ordenamiento territorial obedecen a intereses privados, como proyectos comerciales y residenciales, lo que viene acompañado de infraestructura urbana, como carreteras, viviendas y redes de servicios básicos, convirtiéndose en atractivos para los inversores (Noorloos y Kloosterboer, 2017). Esto, sin duda alguna, conduce a la 
instalación de asentamientos informales por cuanto los expropiados son trasladados desde lugares céntricos hacia las periferias, lo que deviene en la creación de nuevas zonas de riesgo, que con el pasar de los tiempos llegan a convertirse en grandes poblados, dificultando los procesos de mitigación y reducción de riesgos.

\section{El apego a la vivienda y al lugar de origen}

La vivienda es un elemento constituyente del hábitat en donde se desenvuelven las tramas de la vida; por ello, es preciso referirla como sostén para el desarrollo humano, tal y como lo afirma Chardon y Hurtado (2012); para ello, deben cumplir con condiciones dignas y equitativas de permanencia y uso del espacio, según lo manifiesta Mejía-Escalante (2012). Es decir, la vivienda debe tener acceso sostenible a servicios urbanos como agua potable, energía, saneamiento e higiene.

Orozco \& Guzmán (2015) revelan que el suelo más barato se encuentra donde existe una menor cantidad de servicios urbanos, también llamados servicios básicos. Por esta razón, muchos proyectos habitacionales se localizan en zonas periféricas, distantes de centros de comercio, educación o salud, necesarios para el desarrollo; todo obedece a la lógica por disminuir costos del promotor inmobiliario, lo que sin embargo afectará a la municipalidad de turno, que deberá construir infraestructura complementaria, para cubrir la demanda de servicios ciudadanos en la nueva localidad.

Uno de los problemas ligados al reasentamiento consiste en los traslados a grandes distancias, lo que dificulta la adaptación a las nuevas condiciones (Gomersall, 2018); por ello, Ramos (2018) precisa que los gobiernos deben ejecutar iniciativas que promuevan la inclusión de la población desplazada.

La tarea para los desplazados se complica más aún cuando deben construir relaciones con su nuevo territorio, muchas veces en condiciones precarias, perdiendo sus actividades productivas y su red de relaciones sociales (Chenut \& Ocampo, 2014), dado que una parte significativa de los hogares de bajos ingresos utiliza su vivienda como lugar para emprender actividades económicas, haciendo de esta una unidad de producción económica (Barreto, Benítez y Puntel, 2015). Fainstein, (2018) comenta que las viviendas nuevas no poseen estos espacios, lo cual deja sin labores económicas a muchas familias. Todo ello, en fin, nos lleva a repensar si los reasentamientos son la solución a estos conflictos de hábitat.

Berroeta y otros (2017) concluyen que cuando el reasentamiento por riesgos sea la solución más digna para garantizar la vivienda adecuada, hay que considerar el respeto, la protección y la realización del apego, es decir, garantizar la seguridad de los habitantes y promover sus vínculos con el territorio (Carvalho y Cornejo, 2018).

Osorio, Maya \& Rojas (2015) aportan a este postulado, proponiendo que la conformación de una nueva territorialidad incluyente debe brindar oportunidades para recomponer las vidas de los pobladores. Estos migrantes se enfrentan a la creación de nuevos vínculos con otros individuos y con el territorio, dejando huellas y vacios en sus lugares de origen, mientras que, en su travesía, van transformando su cultura, costumbres y comportamientos.

Por último, Morales et al. (2017) estudian el retorno al hábitat de origen por la dificultad de apropiarse y apegarse a la vivienda recibida. Esto ocurre porque no se encuentra la concepción de buena vida, hogar, calma y seguridad. Es por ello que el reasentamiento debe ser asumido como una medida de carácter excepcional, como parte de las estrate- gias de gestión integral del riesgo (Castro \& Vélez, 2018), y no como mero mecanismo de ordenamiento territorial. Para ello, se debe propender a una verdadera reinserción económica, social y ambiental.

\section{El Reasentamiento como mecanismo de gestión de riesgos}

La planificación del reasentamiento es una estrategia popular en todo el mundo: Esta sirve para organizar a las poblaciones desplazadas durante la elaboración de los objetivos políticos-económicos de los países (Gomersall, 2018).

En este sentido, cabe entender que después de los desastres socionaturales las relaciones entre vecinos se transforman, volviéndose más individualistas y defensivos (Berroeta, Carvalho \& Di Masso, 2016).

Se calcula que, hacia la primera mitad de 2019, hubo alrededor de 10.8 millones de nuevos desplazamientos en todo el mundo, de los cuales, siete millones fueron provocados por desastres (Ramírez, 2018); esto hace reflexionar acerca de la importancia de incluir el enfoque de gestión de riesgos en las políticas de ordenamiento territorial, de manera que ello permita mitigar y reducir el impacto de los desastres.

Referente al reasentamiento, Velásquez (2016) manifiesta que "se considera un instrumento de gestión de suelo por demandar nuevo suelo y modificar las características de su uso actual, con el fin de modificar las condiciones de vulnerabilidad física, específicamente la exposición" (p.8). Sin embargo, la modificación de la vulnerabilidad a través del reasentamiento propicia la creación o modificación de otros tipos de vulnerabilidad, como afirma Castro (2017), puesto que las familias afectadas no reciben atención psicológica para enfrentar los efectos del desarraigo mientras que obtienen pagos írrisorios por las expropiaciones.

Takano (2018) al respecto aporta que este reasentamiento involuntario tiende a intensificar la vulnerabilidad social por la poca compensación que conduce al empobrecimiento.

Este desplazamiento forzado implica abandonar la comunidad de origen; con ello, se producen efectos negativos en el bienestar psicosocial y la calidad de vida, generando fuertes situaciones de estrés por la ruptura de vínculos sociales con familiares y amigos (Ramos, 2017).

Esto nos permite discernir que el riesgo de desastre es el resultado de procesos históricos del uso y ocupación del territorio y su desarrollo, lo que lleva a que este se distribuya diferencialmente (Correa et al., 2011), concentrándose en poblaciones de bajos ingresos económicos.

Por tanto, este riesgo se acentúa por prácticas antrópicas inadecuadas y exposición a amenazas naturales, tales como la deforestación y la falta de drenajes, lo que hace que el suelo pierda su capacidad de absorción. A pesar de la terminología "desastre natural", Bravo (2017) aclara que "muchos desastres cuyo origen es considerado como "natural", tiene como origen, o está exacerbado por actividades humanas"

El reasentamiento, como mecanismo de gestión de riesgos introduce comunidades a un nuevo entorno construido como solución a largo plazo, lo que en consecuencia redefine el sistema social. La inadaptación ocurre cuando el diseño de la casa, la infraestructura y el nuevo entorno son inapropiados o insuficientes (Sridarran et al., 2018). Duriez (2019) al respecto, señala que en estos casos es importante considerar el contexto socio-histórico del desarrollo de una población, la acción colectiva de los afectados durante su implementación y la dimensión simbólica de toda política pública. 
De lo dicho, Velásquez (2016), citando a Chardon (2008), "sostiene que detrás de todo proceso de reubicación existe una destrucción de su hábitat humano", ya que la construcción de casas no garantiza condiciones dignas de vida, ni el desarrollo integral de las personas. Anja Klopfer (2015) sugiere que el apego al lugar es un factor determinante en estos proyectos de reconstrucción, de manera que debe prevalecer el sentir colectivo en su implementación.

El riesgo de desastre exige la cooperación del gobierno y la comunidad para concertar acciones aceptables y factibles, frente a eventos inciertos. La reubicación se presenta como un instrumento de intervención dentro del OT, la cual permite reducir la vulnerabilidad física: no obstante, las consecuencias de estos procesos pueden generar aumento de la vulnerabilidad social al convertirse en trampas de segregación y exclusión (Briones, 2010).

La falta de planificación territorial y de programas de vivienda para población de bajos recursos conlleva a asentamientos humanos en zonas de alto riesgo; por ello, medidas como el reasentamiento deben estar orientadas a modificar procesos actuales de uso y ocupación del territorio que generan amenazas y vulnerabilidades, a la vez que propicien el medio adecuado para el desarrollo humano en sus dimensiones social, ambiental, económica y cultural.

\section{CONCLUSIONES}

Reasentar una población constituye no solo el traslado de personas a emplazamientos seguros, dotados de vivienda y servicios que, en teoría, debería contribuir a mejorar su calidad de vida. Va más allá. Comprende el desprendimiento por parte de la comunidad de sus vínculos socioambientales con su hábitat de origen, en donde, probablemente, muchos desplazados nacieron y vieron nacer a sus hijos.

Desde los enfoques del ordenamiento territorial y la gestión del riesgo, los reasentamientos resultan pertinentes, pues modifican la localización de la población vulnerable, eliminando la exposición al riesgo, al no existir coincidencia espacio-temporal con la amenaza natural; sin embargo, pueden originarse otros tipos de vulnerabilidad producto de las afectaciones a la economía familiar y al sentido de identidad cultural y tradicional de las personas, además de situaciones de exclusión social y desarraigo, presentes en la mayoría de los casos.

Si bien estos reasentamientos buscan reducir los riesgos de desastres a la vez que permiten implementar programas de uso adecuado del suelo, es necesario destacar que parte considerable de las familias reasentadas sufren efectos sociales y económicos en el proceso, ya que tradicionalmente utilizan su vivienda no sólo como residencia, sino también para actividades económicas informales, como estrategias de sobrevivencia; sin embargo, en muchos emplazamientos, estas actividades se ven reducidas o limitadas por las políticas de utilización de las viviendas o debido a la existencia de vecinos que ocupan esos nichos económicos, lo que afectará a mediano y largo plazo el proceso de adaptación al nuevo entorno.

\section{REFERENCIAS}

Barreto, M., \& Benítez, M., \& Puntel, M. (2015). Vivienda Social y estrategias de sobrevivencia. soluciones adecuadas a partir de un estudio de caso (Resistencia, Argentina, 2013). Revista INVI, 30(84),19-57. https://www.redalyc.org/articulo. oa?id=258/25840411002

Baum, H. (2015). Planning with half a mind: Why planners resist emotion. Planning Theory \& Practice, 16(4), 498-516. D0l: https:// doi.org/10.1080/14649357.2015.1071870

Berroeta, H., Carvalho, L., Di Masso, A., Ossul, M. (2017). Apego al lugar: una aproximación psicoambiental a la vinculación afectiva con el entorno en procesos de reconstrucción del hábitat residencial. Revista INVI, 32(91),113-139. https://www.redalyc.org/ articulo.oa?id=258/25855071005

Berroeta, H., Carvalho, L., \& Di Masso, A., (2016). Significados del espacio público en contextos de transformación por desastres socionaturales. Revista INVI, 31(87),143-170. https://www.redalyc. org/articulo.oa?id=258/25847811005

Bravo, E. (2017). El sismo del 16 de abril en Manabí visto desde la ecología política del desastre. Universitas. Revista de Ciencias Sociales y Humanas, (26), 235-252. https://www.redalyc.org/ articulo.oa?id=4761/476151860010

Briones F. (2010). "Inundados, reubicados y olvidados: Traslado del riesgo de desastres en Motozintla, Chiapas". Revista de ingeniería. (31) 132-144. http://www.scielo.org.co/pdf/ring/n31/n31a14. pdf

Pinto de Carvalho, Laís \& Cornejo, Marcela (2018). Por una aproximación crítica al apego al lugar: una revisiónen contextos de vulneración del derecho a una vivienda adecuada. Athenea Digital, 18(3), e2004. https://doi.org/10.5565/rev/athenea.2004

Castiblanco-Prieto, J., Aguilera-Martínez, F. \& Sarmiento-Valdés, F. (2019). Principios, criterios y propósitos de desarrollo sustentable para la redensificación en contextos urbanos informales. Revista de Arquitectura. 21(1), 21-33. http://dx.doi.org/10.14718/ RevArq.2019.21.1.1209

Castro, E. (2017). Derecho ambiental y gobernanza. La concertación informal de la Administración. Medellín: Universidad de Medellín Ed.

Castro-Buitrago, E., \& Vélez, J. (2018). Procesos de reasentamiento en Colombia: ¿Una medida de adaptación y protección de derechos humanos de las víctimas del cambio climático? Vniversitas (136), 1-10. http://www.scielo.org.co/pdf/vniv/n136/00419060-vniv-136-00020.pdf

Chardon A. (2008). "Reasentamiento y hábitat en zonas urbanas, una reflexión en Manizales". Cuaderno de vivienda y urbanismo. 1(2). https://revistas.javeriana.edu.co/index.php/cvyu/article/ view/5491

Chardon, A. \& Hurtado, J. (2012). Vivienda social y reasentamiento, una visión crítica desde el hábitat. Manizales: Universidad Nacional de Colombia. http://www.bdigital.unal.edu.co/9432/7/Nivienda\%20Social\%20y\%20Reasentamiento.pdf

Chenut P. \& Ocampo M. (2014). Reasentamiento tras el desplazamiento forzado: dos comunidades étnicas en Colombia. Iztapalapa. Revista de ciencias sociales y humanidades, 35(76), 105-137. https://dx.doi.org/10.28928/ri/762014/atc4/chenutcorreap/ ocampopradom

CMMAD. (1988). Nuestro Futuro Común. Madrid: Alianza Editorial.

Codosero, J., Castanho, R., Cabezas, J., \& Naranjo J. (2020). Sustainable valuation of land for development. Adding value with urban planning progress. A Spanish case study. Land Use Policy, 92, 
104456. https://doi.org/10.1016/j.landusepol.2019.104456

Correa, E. Sanahuja, H., \& Ramírez F. (2011). Guía de Reasentamiento para poblaciones en riesgo de desastre. Banco Mundial y el Global Facility for Disaster Reduction and Recovery (GFDRR), 33-46. Washington DC.

Duriez, T. (2019). El desplazamiento forzado intraurbano: una modalidad de movilidad residencial a las coacciones controvertidas. Territorios (40), 245-272. http://dx.doi.org/10.12804/revistas. urosario.

Engelman, R., (2013). Beyond Sustainababble. En Assadourian E. y Prugh T. (editores), Is Sustainability Still Possible? (p.3-18). Washington DC: Island Press

Fainstein, C. (2018). Las relocalizaciones masivas de población en los estudios urbanos. aportes desde la experiencia de un asentamiento informal en la ribera del Riachuelo. Cuaderno Urbano. Espacio, cultura, sociedad, 24(24),93-116. https://www.redalyc.org/articulo.oa?id=3692/369259347005

Galarza, C. (2019). La ciudad intermedia en la escala regional; el caso de Cuenca-Ecuador. (Trabajo de Fin de Máster no publicado). Universidad de Cuenca, Ecuador.

Gomersall, K. (2018). Resettlement practice and the pathway to the urban ideal. Geoforum, 96, 51-60. https://doi.org/10.1016/j. geoforum.2018.07.018

IDMC. (2019). Internal Displacement From January to June 2019. [Fecha de consulta: 24 de mayo de 2020] https://www.internal-displacement.org/sites/default/files/inline-files/2019-mid-year-figures_for\%20website\%20upload. pdf

Iturralde, C. (2019). Los paradigmas del desarrollo y su evolución: del enfoque económico al multidisciplinario. Retos Revista de Ciencias de la Administración y Economía, 9(17), 7-23. https://doi. org/10.17163/ret.n17.2019.01

Klopfer, A. (2015). "Choosing to Stay": Hurricane Katrina Narratives and the History of Claiming Place-Knowledge in New Orleans. Journal of Urban History, 1-25. https://doi. org/10.1177/0096144215576332

López, F. (2011). El análisis de contenido como método de investigación. Clave PedagóGica, (4) 167-179.

Mejía-Escalante, M. (2012). Habitabilidad en la vivienda social en edificios para población reasentada: El caso de Medellín, CoIombia. EURE (Santiago), 38(114), 203-227. https://dx.doi. org/10.4067/S0250-71612012000200008

Morales, R., Besoain, C., Soto, A., Carvalho, L., Hidalgo, K., Fernández, I., \& Bernal Santibáñez, V. (2017). Retorno al campamento: resistencia y melancolía en los márgenes de la ciudad formal. Revista INVI, 32(90), 51-75. https://doi.org/10.4067/S071883582017000200051

Nikuze, A., Sliuzas, R., Flacke, J., \& van Maarseveen, M. (2019). Livelihood impacts of displacement and resettlement on informal households - A case study from Kigali, Rwanda. Habitat International, 86, 38-47. https://doi.org/10.1016/j.habitatint.2019.02.006

Noorloos, F., Kloosterboer, M. (2017) Africa 's new cities: The contested future of urbanisation. Urban Studies, 55 (6) (2018), pp. 1223$1241 \mathrm{https}: / /$ doi.org/10.1177/0042098017700574

ONU-Habitat. (2016). Urbanización y desarrollo: futuros emergentes. Reporte Ciudades del Mundo Nairobi: Centro Urbano ed.

Orozco, I. \& Guzmán, S. (2015). Reflexiones sobre la habitabilidad de la vivienda social. El Área Metropolitana Centro Occidente, CoIombia. Revista Bitácora Urbano Territorial, 25(1),27-35. https:// www.redalyc.org/articulo.oa?id=748/74841604003

Orsini, C. A., Danús, M. T., \& Tricio, J. A. (2019). La importancia de la educación interprofesional en la enseñanza de la odontología: una revisión sistemática exploratoria analizando el dónde, el porqué y el cómo. Educación Médica, 20, 152-164.

Osorio, A. (2017). Urbanismo, Reasentamiento de Población y Vivienda Adecuada: desafíos para la defensa de los derechos humanos en los territorios. Ratio Juris, 12(24),61-86. https://doi. org/10.24142/raju.v12n24a3

Osorio-Campillo, H., Maya-Sierra, T. \& Rojas-Sánchez, E. (2015). Territorios y migraciones. Territorialidades en transformación. Revista Bitácora Urbano Territorial, 25(1),93-102. https://www.redalyc. org/articulo.oa?id=748/74841604013

Ramírez, I. (2018). El Registro Nacional para las Personas Desplazadas: características y limitaciones. Revista Mexicana de Sociología, 80(3),675-702. https://www.redalyc.org/articulo. oa?id=321/32158229007

Ramos, I. (2018). Desplazamiento forzado y adaptación al contexto de destino: el caso de Barranquilla. Perfiles latinoamericanos, 26(51), 301-328. https://dx.doi.org/10.18504/pl2651-0122018

Ramos, I. (2017). Dinámicas comunitarias en desplazados y no desplazados residentes en zonas de exclusión social en Barranquilla (Colombia). Revista de Estudios Sociales, (60), 49-61. https:// dx.doi.org/10.7440/res60.2017.04

Sridarran, P., Keraminiyage, K., \& Amaratunga, D. (2018). Enablers and barriers of adapting post-disaster resettlements. Procedia Engineering, 212, 125-132. https://doi.org/10.1016/j. proeng.2018.01.017

Takano, G. (2018). Resettlement resulting from the development of an infrastructure megaproject in Lima, Peru. An analysis on housing habitability and social relations. Revista INVI, 33(94), 135-159. https://dx.doi.org/10.4067/S0718-83582018000300135

Velásquez, J. (2016). El proceso de Reasentamiento en la modificación de la vulnerabilidad física y social en las zonas de amenaza volcánica alta en los sectores urbanos de Pasto y la Florida, departamento de Nariño, Colombia (Trabajo de Fin de Máster no publicado). FLACSO, Ecuador. 